\title{
Modeling Solar Converters for Harmonic and Resonance Studies
}

\author{
M. Pielahn, K. Mudunkotuwa, and D. Muthumuni
}

\begin{abstract}
Solar and renewable plants are increasingly being added to national grids. One technical drawback of renewable generators is that they tend to cause a significant harmonic distortion of the current and voltage waveforms. The harmonic distortions are amplified when the natural resonances of the grid interact with the injected harmonics from the power electronic based PV generation. A sustained harmonic distortion can lead to equipment failure, reduced plant output or a complete plant shutdown. Therefore, it is important to study harmonic interactions and resonance issues using fully detailed EMT models before the commissioning of a renewable plant. This paper outlines important features of modelling solar converters for the use of harmonic and resonance studies.
\end{abstract}

Index Terms-EMT modeling, aggregation.

\section{INTRODUCTION}

$\mathrm{I}_{\mathrm{i}}^{\mathrm{N}}$ $\mathrm{N}$ recent years, solar and renewable generators are increasingly being connected to the national grid. Renewable generators tend to cause higher levels of harmonic distortion due to the power electronic based converters injecting harmonic currents in to the grid [1]. Therefore, with an increased number of renewable generators active in the gird the need to perform detailed studies is growing.

Under some circumstances harmonic resonance can be an issue when the harmonics are sustained and potentially amplified. This can happen because of the interaction of the renewable converter current injections with the grid resonance points. These interactions can happen in the sub-synchronous as well as the super-synchronous frequency range.

Sustained harmonic distortion may create an over-voltage that could exceed acceptable harmonic distortion levels. This could trip the protection or even damage the equipment, and interrupt the operation of the plant [2]. Hence, it is important to study harmonic and resonance issues using detailed EMT models before the actual plant is commissioned.

This paper presents a method to model solar farms for harmonic and resonance studies. First, the implementation of detailed converter models will be discussed. Next, aggregation of PV collector networks is outlined, followed by a discussion of representing the AC grid for such studies.

Manuscript received March 30, 2017; revised April 4, May 6, 2017; accepted April 18, 2017.

M. Pielahn, K. Mudunkotuwa, and D. Muthumuni are with Manitoba HVDC Research Center, Winnipeg, Manitoba, Canada R3P1A3 (e-mail: mpielahn@hvdc.ca; ckumara@hvdc.ca; dharshana@hvdc.ca).
The discussion continues with passive and dynamic impedance scans. The impedance scan results are useful indicators of impending operational issues. Lastly, important study scenarios are discussed that should be reviewed in order for a study engineer to analyze any harmonic and resonance issues.

\section{Detailed CONVERTER Models}

Typically harmonic resonance issues arise due to the interaction between the converter, the PV farm collector cable system and the local grid within a close proximity of the solar farm. For harmonic and resonance type studies in EMT, it is advised that detailed vendor models be used such that the actual controllers are properly modelled in the simulation. This is in view of the fact that the control actions have an impact on the harmonics injected by the PV converters.

Generic solar models may not capture the necessary accurate information that is used to decide whether a particular solar farm does or does not have any harmonic or resonance issues. Detailed solar converter models should include accurate PLLs, $d q$ decoupling controllers, filters, protections schemes and fault ride-through options. The main components of a PV plant model is shown in

Fig. 1.

\section{Aggregation of Collector Networks}

Performing simulations with detailed EMT type converter models can be computationally demanding, especially if the solar plant consists of many inverters and a large collector network. Therefore, an EMT simulation may take on the order of several hours. Further, harmonic and resonance studies require that many different operating conditions are to be studied in order to identify sensitive parameter and worst case operational conditions. Thus, in total a detailed harmonic and resonance study may demand a simulation time that is not practical or economical.

In recent years an industrial trend of collector network aggregation has emerged. This allows for the larger collector network to be represented by an equivalent cable that portrays the characteristics of the actual collector network within the frequency bandwidth of interest. If the aggregation is done properly, the important details contributing to potential harmonic and resonance issues are preserved, while at the same time reducing the computational burden and speeding up the simulation.

In order to aggregate a collector network, an impedance scan must be performed, looking from the point of 
interconnection (POI) towards the converters. Such a scan will reveal any series and parallel resonance points. Aggregation usually only preserves the details of a single series (or parallel) resonance point as it is difficult to incorporate two or more peaks in the aggregated model. Therefore, the aggregated model only covers a frequency band of interest, usually centered on the series resonance point.

Collector networks usually are comprised of multiple feeders, which contain a daisy chain of PV inverters (typically that includes the pad mount transformers) and cables. These daisy chains may consist of PV units only in series, or a combination of series and parallel sub-daisy chains. A hypothetical situation of a collector network with two such feeders is shown in

Fig. 2.

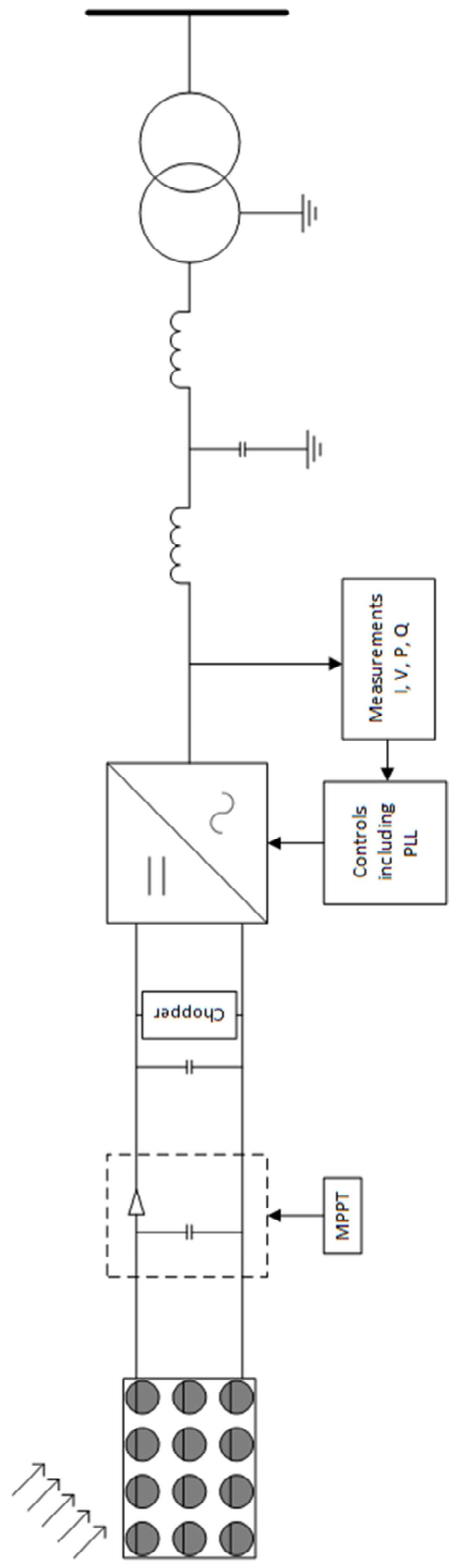

Fig. 1. Schematic of a detailed PV model.

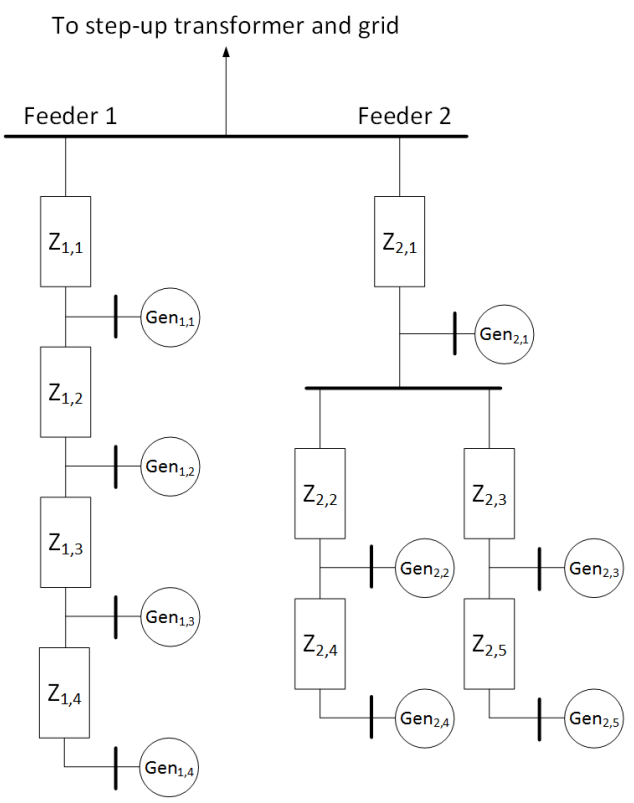

Fig. 2: Hypothetical collector network with two feeders.

Methods to aggregate feeders of a collector system as shown in

Fig. 2 have been reported in literature [3, 4]. In [3] a detailed equivalencing method for both series and parallel units has been shown. Both [3] and [4] outline equivalencing methods and equations. A sound theoretical explanation is presented for [3]. An extended curve fitting method is proposed in [4] to obtain a closer match of network resonance points.

This curve fitting method is applied after obtaining the preliminary equivalent parameters using the listed equations of [4]. This allows for the final aggregated model to accurately contain the resonance point in the frequency band of interest by fine-tuning the resonance peak. A typical impedance plot of a collector system cables is shown in

Fig. 3.

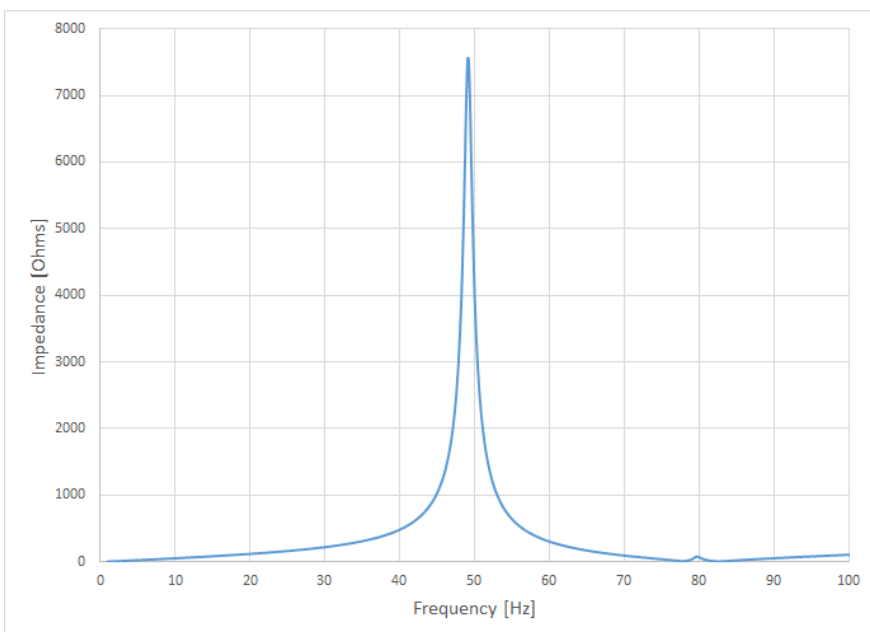

Fig. 3. Passive impedance scan of an example PV collector network. 


\section{EQUIVALENCING OF THE GRID}

At the POI the grid should be modelled in as much detail as possible to capture the network frequency characteristics (impedances as seen at different frequencies). Typically, this requires that the network be represented in detail up to around two to three buses from the POI.

All transmission lines, generators, loads or other equipment outside this area can be modelled using Thévenin equivalent voltage sources. The range in which to include full details of the network is defined by the study engineer after carefully assessing the frequency response of the full network.

The frequency response of the reduced network should closely match that of the original full network. For example, a generator that is far from the POI is unlikely to impact the network frequency characteristics as seen from the POI. Therefore, a study engineer assesses how far away the details of the network need to be included in the reduced grid.

Special attention should be made for series compensation lines as they may strongly contribute to the natural resonance points of the network. Series compensation lines are considered when selecting study scenarios. Network conditions that may result in a nearby series compensated line to be radially, or near radially, connected to the solar farm should be given due consideration.

\section{PASSIVE AND DYNAMIC IMPEDANCE SCANS}

The network impedance looking in to the AC system from the POI as well as the impedance of the PV farm looking from the POI is useful indicators of potential harmonic and resonance issues. The impedance of the PV system is dynamic in the sense that power electronic devices, control systems actions can have a significant impact on this 'apparent impedance'. The real part of the 'apparent impedance' (dynamic resistance) is an indication of damping that the PV installation will provide to specific harmonic currents that will flow in to the system following a disturbance. In order to obtain a dynamic resistance spectrum a dynamic scan needs to be performed.

On the other hand, looking into the grid from the POI gives the passive impedance of the network. A graphical representation of the 'measuring point' (in this case the POI) is shown in Fig. 4.
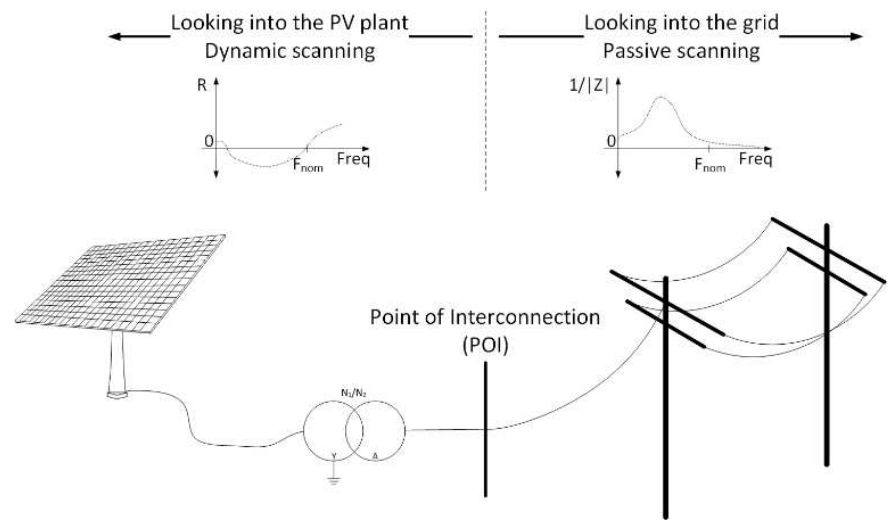

Fig. 4. Dynamic and passive scanning into the PV plant and the grid, respectively.
A passive impedance scan is easily obtained in PSCAD $^{\mathrm{TM}}$, using a standard Master Library component, allowing for the user to simply scan any passive network. An example of a typical impedance scan of a network is shown in

Fig. 5.

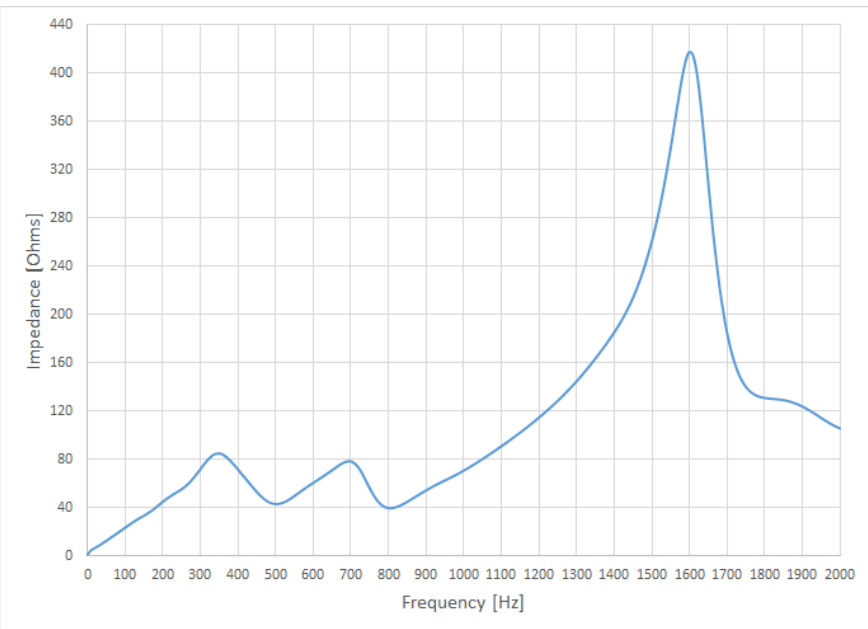

Fig. 5. Passive impedance scan of an example network.

However, a dynamic scan is more involved and should be done with care. For full details the reader is referred to $[5,6]$. The PV plant model may be isolated from the grid for this exercise, with an ideal voltage (at the rated voltage) representing the AC grid side. The PV plant should be set to run at the expected steady state condition. A 'small' harmonic voltage is injected to this test simulation as depicted in Fig. 6.

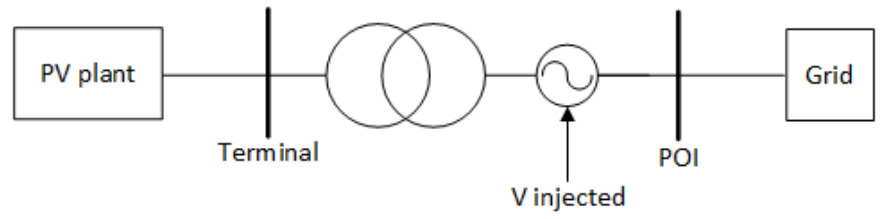

Fig. 6. System overview with small harmonic voltage injection.

The amplitude of the injected voltage should be large as to disturb the system enough in order to make meaningful measurements. However, too large of an amplitude may cause the overall PV system response to deviate significantly from its operating steady state. This should also be avoided.

When the simulation has reached steady state, a voltage and current measurement can be made at the terminal (see Fig. 6). Using these quantities, the dynamic impedance can be determined corresponding to the particular frequency of the injected voltage. In order to obtain a spectrum, the procedure can be repeated for the harmonics of interest.

An example of the impedance (resistance) profile of a solar converter developed in PSCAD is shown

Fig. 7. The negative 'dynamic resistance' is an indication of negative damping. The PV system in such a case will amplify transient currents of this frequency range following a disturbance and cause instability.

The passive scan of the AC system along with the dynamic 
scan of the converter can be compared to identify potential interaction issues.

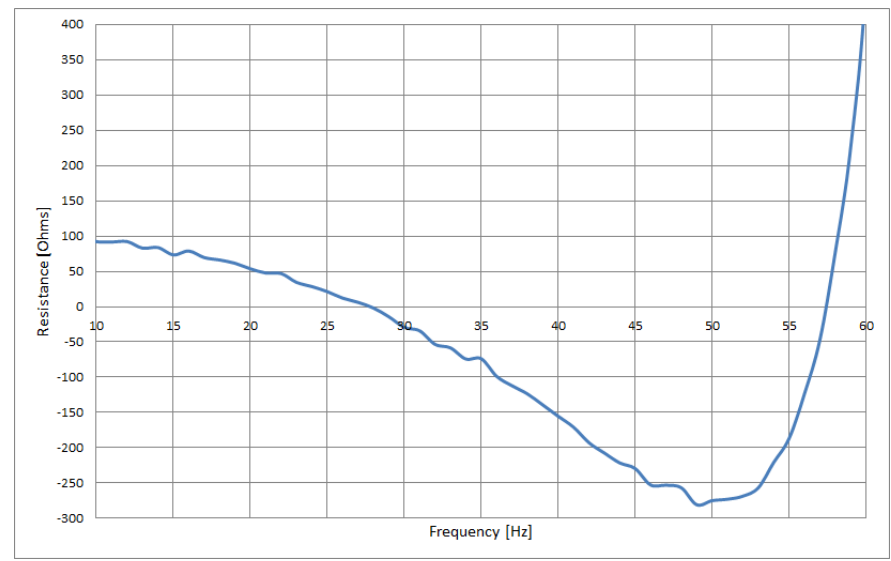

Fig. 7. Dynamic impedance scan of an example aggregated solar farm network.

If the passive scan of the grid show a resonance point within, or close to, the range of frequencies for which the dynamic resistance is negative, then a potential harmonic and resonance issues are a possibility. Such scenarios are identified as candidates for further detailed EMT transient simulations.

\section{STUDY SCENARIOS}

Resonance and harmonic issues may be triggered during specific operating conditions of the AC system and the PV plant. Thus, the study engineer should identify credible operating scenarios for the analysis. The scenarios may be categorized as follows.

1) PV plant operating condition (power level, voltage control settings); and

2) AC system operating conditions (equipment outages, etc.).

Different types of disturbances should be studied for each selected scenario. This includes different fault types and locations.

One of the most important disturbances to study in references to harmonic and resonance issues is a fault, or a set of faults, that cause the PV plant to be radial, or near radial, with any series compensation lines. Series compensation lines can cause sub-harmonic currents to flow in to the PV plant leading to instabilities.

A PV plant may operate between a range of realizable power output levels, and several of them should be checked for resonance and harmonic conditions. This is in view of the fact that the converter may have different pre-defined control options and settings depending on the operating condition.

\section{CONCLUSIONS}

Important features of a solar PV model used in harmonic and resonance studies are outlined. First the significance of detailed EMT models is discussed. Generic solar models may not capture critical features needed to accurately study harmonic and resonance issues. A discussion that outlines the methodology to aggregate the collector network of solar plants is presented. First the collector network is equivalence based on mathematical approximations, and then the frequency profile is curve fitted to a match the original resonance peak.

The passive and dynamic frequency scanning methods and their significance is discussed with appropriate references to previous work outlining details. Typical network frequency scans and dynamic frequency scans of PV plants are presented. The two scans are compared and potential resonances issues are identified.

\section{REFERENCES}

[1] R. C. Dugan, S. Santoso, M. F. McGranaghan, and H. W. Beaty, "Electrical System Power Quanlity", $2^{\text {nd }}$ Edition, McGraw-Hill, 2002.

[2] R. K. Varma, S. A. Rahman, T. Vanderheide, M. D. N. Dang, "Harmonic Impact of a 20-MW PV Solar Farm on a Utility Distribution Network", IEEE Power and Energy Technology Systems, Vol. 3, pp. 89-98, 2016

[3] E. Muljadi, et al., "Equivalencing the Collector System of a Large Wind Power Plant", IEEE Power Engineering Society General Meeting, 2006.

[4] M. Pielahn, et al., "Efficient EMT modeling approach to studying resonance phenomenon in PV and wind energy systems", International Conference on Power Systems Transients, 2015.

[5] M. Sahni, D. Muthumuni, B. Badrzadeh, A. Gole, A. Kulkarni, "Advanced screening techniques for Sub-Synchronous Interaction in wind farms," in 2012 Transmission and Distribution Conference and Exposition (PES T\&D), pp. 1-9.

[6] B. Badrzadeh, M. Sahni, D. Muthumuni, Y. Zhou and A. Gole, "Subsynchronous Interaction in Wind Power Plants-Part 1: Study Tools and Techniques," in 2012 IEEE Power and Energy Society General Meeting, pp. 1-9.

M. Pielahn, EIT received the B.Sc. degree from the University of Winnipeg (Honours-2011) and the M.Sc. degree from the University of Manitoba (2014). He has been involved in a variety of PSCAD and PSS/E related projects, including EMT simulations studies for HVDC, wind and solar projects, SSCI studies, and custom component development. Mathias. He has experience with software tools and programming languages including PSCAD, MatLab/Simulink, Fortran, PSS/E and DIgSILENT.

Kumara Mudunkouwage, P. Eng. received the B.Sc. degree from University of Moratuwa (Sri Lanka) in 2002 and the M.Sc. degree from University of Manitoba in 2011. He has been working at the Manitoba HVDC Research Centre as a Technical Support \& Consulting Engineer since 2011. Kumara has extensive work experience in power system modeling and simulation, particularly in the areas of power system planning and operational studies. He has been involved in various consulting projects at the Manitoba HVDC Research Centre, including HVDC and generation interconnections, wind and solar integration, and analysis of the power quality and harmonics issues. In addition, he has been involved in various projects to develop software tools for engineering studies.

Dr. Dharshana Muthumuni, P. Eng. is the Managing Director of the Manitoba HVDC Research Centre, a division of Manitoba Hydro International. He has over 20 years of experience in engineering studies using a variety of simulation products, including PSCAD ${ }^{\mathrm{TM}}$ and PSS/E. His expertise is regularly sought out by clients around the world for his strong and wide ranging technical knowledge on power system behavior, model development, and simulation studies. He has lead the technical team to solve challenging problems, including HVDC and generation interconnections, wind integration into weak grids, FACTS based solutions, SSR screening techniques, and power quality and harmonics. He has worked extensively and closely with equipment vendors to develop simulation models and techniques to address difficult interconnection problems. He has developed many customized models and simulations techniques for specific studies, including working closely with equipment vendors to address their simulation study requirements. In addition, he has been a key developer of the PSCAD simulation tool and has conducted training workshops on a variety of power system topics for our global clients. In his current position, Dharshana is responsible for overseeing the development and deployment of all MHRC's products and services including PSCAD ${ }^{\mathrm{TM}}$, HVDC Line Fault Locator System, Engineering Consulting, Training, and Research \& Development. 